\title{
Deep Epigastric Lymph Nodes Implication in Patients' Recurrence Pattern After Cytoreductive Surgery in Colorectal Peritoneal Metastases
}

\author{
Antoine El Asmar ${ }^{1}\left[\right.$ [ Michael Vouche ${ }^{2} \cdot$ Maria Gomez Galdon $^{3} \cdot$ Maria Antonietta Bali $^{2} \cdot$ Francesco Sclafani $^{4}$. \\ Vincent Donckier $^{1} \cdot$ Gabriel Liberale $^{1}$
}

Received: 21 October 2021 / Accepted: 30 November 2021

(c) The Society for Surgery of the Alimentary Tract 2021

Keywords Peritoneal carcinomatosis $\cdot$ Colorectal cancer $\cdot$ Lymphatic system $\cdot$ Cytoreductive surgery $\cdot$ Recurrence

\section{Introduction}

The peritoneum is a common site for metastasis from various gastrointestinal tumors. Approximately $7 \%$ of colorectal cancers (CRC) present with peritoneal metastases (PM), and up to $19 \%$ of CRC develop PM, even after curative surgery. ${ }^{1,2}$ Furthermore, recurrence is quite common for patients with $\mathrm{PM}$, after cytoreductive surgery, with or without hyperthermic intraperitoneal chemotherapy (CRS \pm HIPEC). As demonstrated in a recent study, the deep epigastric lymph node (DELN), at the level of the inferior epigastric artery, may represent a possible dissemination pathway for PMCRC, from the peritoneum to the extraperitoneal compartment. ${ }^{3}$ The objective of our study was to evaluate the lymphatic pattern of recurrence, focusing on the previously overlooked DELN, in PMCRC patients treated with CRS.

Paper Presented in Belgian Surgical Week 2021, $22^{\text {nd }}$ Edition, Sept 09-11, Antwerp.

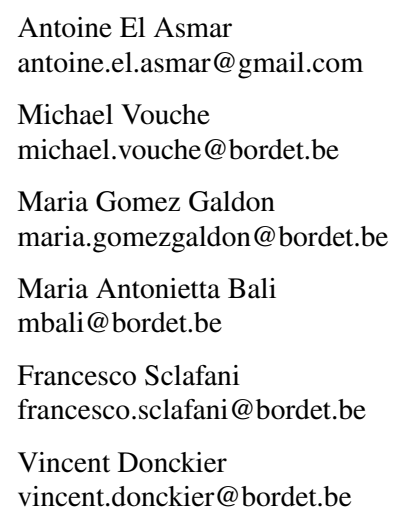

\section{Patients and Methods}

This retrospective study included patients treated for PMCRC with curative intent by CRS \pm HIPEC (open technique), between 2012 and 2018, at Institut Jules Bordet, and who presented with disease recurrence. Ethical committee approval was obtained (CE3281). Two senior radiologists and one surgeon reviewed all patients' CT scans and identified recurrent metastatic sites, including the previously overlooked DELNs.

\section{Results}

Eighty patients had PMCRC, with 60 (75\%) diagnosed with a recurrence at the time of this study. Ten were excluded for not having an available CT scan. Among the 50 included
Gabriel Liberale

gabriel.liberale@bordet.be

1 Department of Surgical Oncology, Institut Jules Bordet, Université Libre de Bruxelles (ULB), Rue Meylemeersch 90, 1070 Brussels, Belgium

2 Department of Radiology, Institut Jules Bordet, Université Libre de Bruxelles (ULB), Brussels, Belgium

3 Department of Pathology, Institut Jules Bordet, Université Libre de Bruxelles (ULB), Brussels, Belgium

4 Department of Oncology, Institut Jules Bordet, Université Libre de Bruxelles (ULB), Brussels, Belgium 


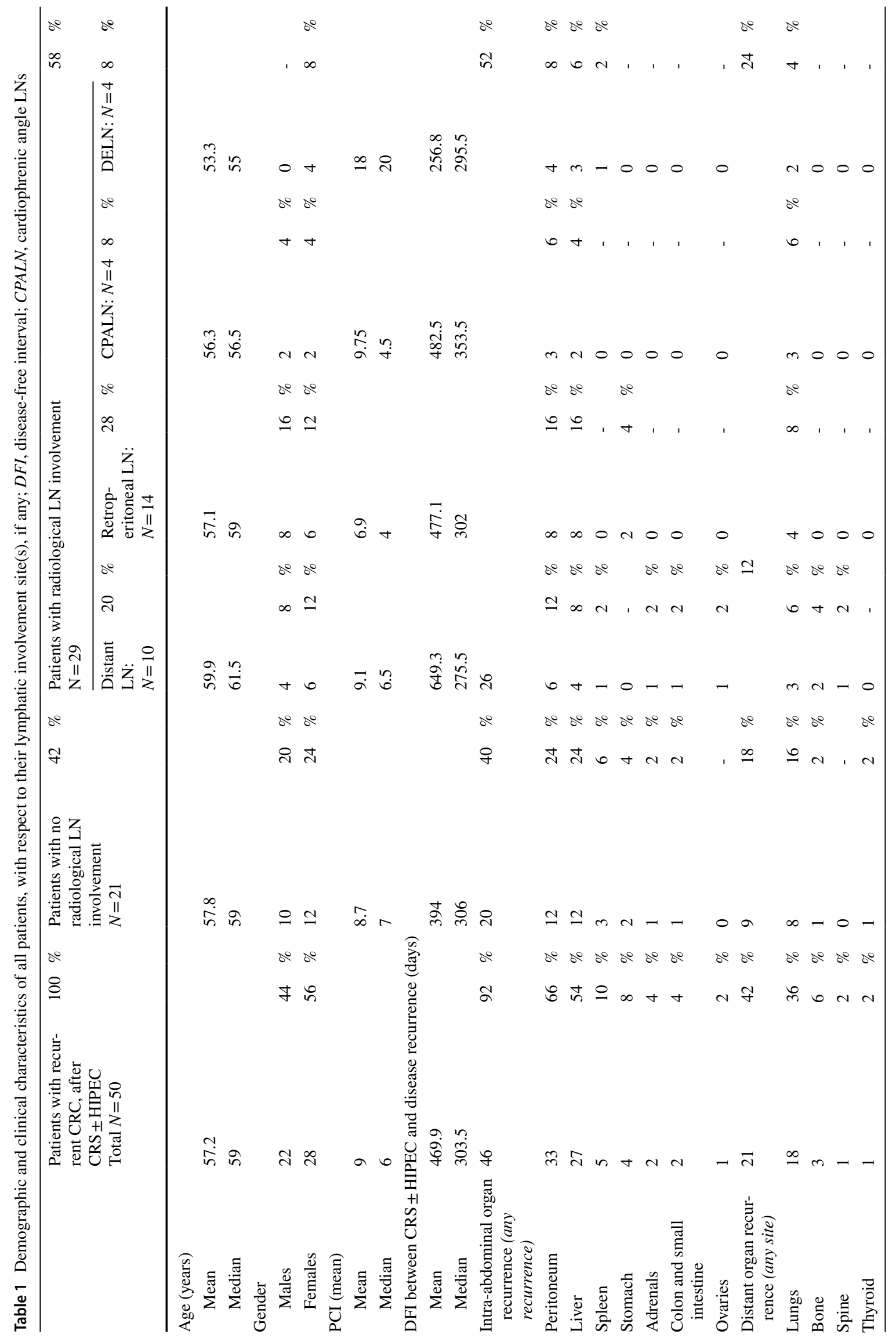



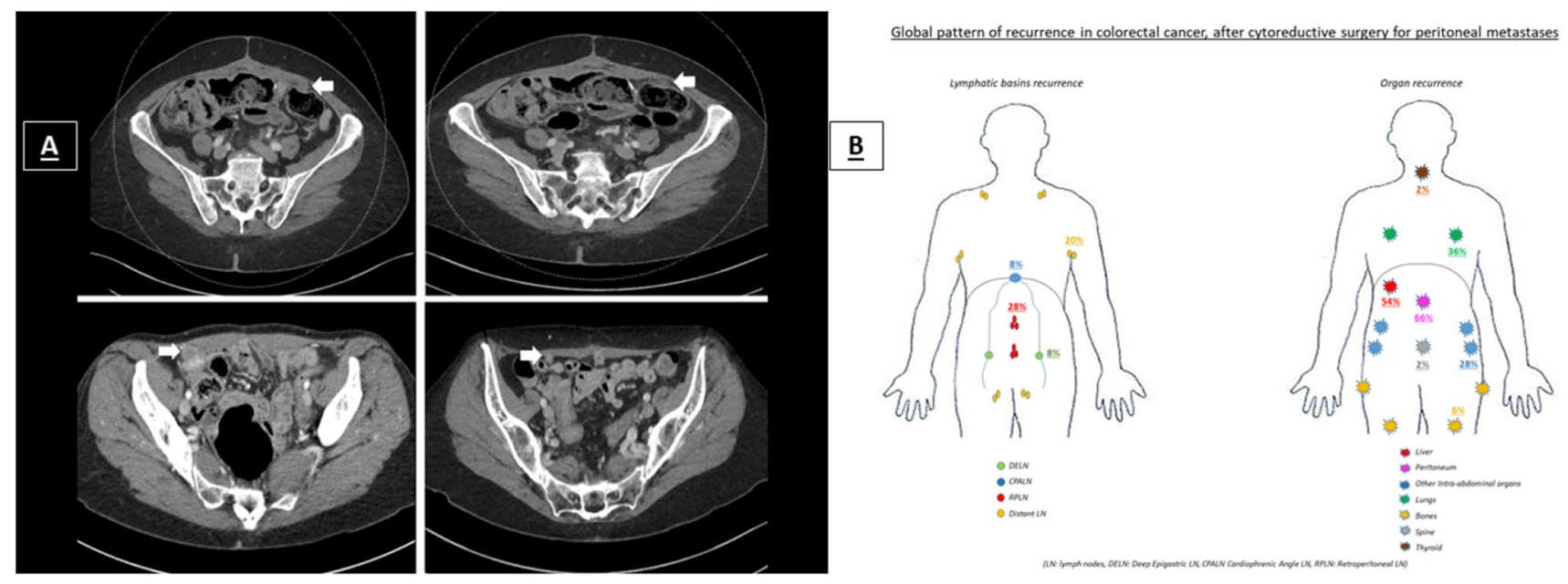

Fig. 1 A Enlarged deep epigastric lymph node detected (white arrow), at the level of the inferior epigastric arteries. B Global pattern of recurrence after CRS for PMCRC

patients, 29 (58\%) had radiological LN involvement. Table 1 summarizes the characteristics of these patients and the different lymphatic basins involved. Four patients (8\%) had a previously overlooked enlarged DELN (Fig. 1). Interestingly, these patients had a higher peritoneal carcinomatosis index (PCI) at initial CRS (before recurrence) than all the other groups.

Furthermore, when comparing the site of recurrence between different groups of patients, we found that $100 \%$ of those who had DELN involvement had PM recurrences, versus 75\%, 57\%, 60\%, and 57\% for the "cardiophrenic LNs," "retroperitoneal LNs," "distant LNs," and "no LNs" groups, respectively.

\section{Discussion}

As a main result of our study, we found that the incidence of radiologic involvement of the DELNs was $8 \%$. We observed that $100 \%$ of our patients with radiologically positive DELNs had peritoneal disease involvement, which enhances the tight relationship between this lymphatic basin and the peritoneum specifically. Moreover, patients with suspicious DELNs had a higher PCI $($ mean $=18)$ than patients in other groups suggesting that this pathway is essentially involved in patients with high peritoneal tumor burden.

Whether the relationship between peritoneum and DELNs depends on the origin of the primary tumor, the PCI score, the main affected zones, or other clinicopathological factors is yet to be determined. However, we were able to show that such a relationship exists and that it should be further investigated to determine the prognostic and clinical relevance of the DELNs in the context of PM.
The weaknesses of this study are its retrospective design, the lack of pathological confirmation of the suspected LNs, and the study's small sample size. However, these observations clearly suggest a potential role of the DELNs in the systemic dissemination process of PMCRC.

\section{Conclusion}

Deep epigastric lymph nodes could represent an important lymphatic drainage pathway for tumors involving the peritoneum, as a relay between the abdominal, and the systemic compartments. This suggests that staging of these specific lymph nodes could be critical in patients with PMCRC, for the therapeutic decision in those who are candidates for CRS \pm HIPEC, and for perioperative management and follow-up.

Author Contribution Antoine El Asmar: conception, design of the work; acquisition, analysis; drafting the work; gave his final approval; and agrees to be accountable for all aspects of the work.

Michael Vouche: design of the work; analysis; revision; gave his final approval; and agrees to be accountable for all aspects of the work.

Maria Gomez Galdon: conception; acquisition; revision; gave her final approval; and agrees to be accountable for all aspects of the work.

Maria Antonietta Bali: conception; analysis; revision; gave her final approval; and agrees to be accountable for all aspects of the work.

Francesco Sclafani: design of the work; acquisition; revision; gave his final approval; and agrees to be accountable for all aspects of the work.

Vincent Donckier: conception; analysis; revision; gave his final approval; and agrees to be accountable for all aspects of the work.

Gabriel Liberale: conception, design of the work; analysis; revision; gave his final approval; and agrees to be accountable for all aspects of the work. 


\section{Declarations}

Conflict of Interest The authors declare no competing interests.

\section{References}

1. M. Barat et al., "The presence of cardiophrenic angle lymph nodes is not an indicator of peritoneal carcinomatosis from colorectal cancer on MDCT: Results of a case-control study," 2015, https:// doi.org/10.1016/j.ejso.2015.11.011.
2. S. Prader et al., "Surgical management of cardiophrenic lymph nodes in patients with advanced ovarian cancer," 2016, https:// doi.org/10.1016/j.ygyno.2016.03.012.

3. [3]A. El Asmar, I. Veys, D. Larsimont, V. Donckier, and G. Liberale, "Inferior epigastric artery lymph nodes: A pathway for systemic dissemination from peritoneal carcinomatosis?," J. Surg. Oncol., vol. 123, no. 1, pp. 311-314, 2021, https://doi.org/10. $1002 /$ jso.26269.

Publisher's Note Springer Nature remains neutral with regard to jurisdictional claims in published maps and institutional affiliations. 